TM-2199

\title{
COUPLED-BUNCH INSTABILITIES OF THE TEVATRON AT RUN II
}

\author{
K.Y. Ng \\ Fermi National Accelerator Laboratory,* P.O. Box 500, Batavia, IL 60510
}

(January 17, 2003)

\begin{abstract}
The longitudinal and transverse coupled-bunch instabilities of the Tevatron at Run II are addressed in two scenarios. The first scenario corresponds to the present Run II condition: 36 proton bunches on 36 antiprotons. Each proton bunch contains $1.7 \times 10^{11}$ particles with a rms bunch length $60 \mathrm{~cm}$. The second scenario is for the future upgrade when there are 108 proton bunches colliding with 108 antiproton bunches. Each proton bunch contains $2.7 \times 10^{11}$ particles with a rms bunch length $50 \mathrm{~cm}$. Our analysis shows that the growth rates of transverse coupled-bunch instabilities are slow and will be damped by a small betatron tune spread. On the other hand, growth rates of longitudinal coupled-bunch instabilities will be fast especially for the 108-by-108 scenario.
\end{abstract}

*Operated by the Universities Research Association, Inc., under contract with the U.S. Department of Energy. 


\section{LONGITUDINAL COUPLED-BUNCH INSTABILITIES}

\subsection{SACHERER'S FORMULAS}

The long-range wake left by the higher-order resonant modes of the rf cavities may couple the longitudinal motions of the bunches in the Tevatron. Assuming $M$ bunches of equal intensity equally spaced in the ring, there are $\mu=0,1, \cdots, M_{s}-1$ modes of oscillations in which the center-of-mass of a bunch leads its predecessor by the synchrotron phase $2 \pi \mu / M_{s}$. In addition, an individual bunch in the $\mu$-th coupled-bunch mode can oscillate in the synchrotron phase space about its center-of-mass in such a away that there are $m=1,2, \cdots$ nodes along the bunch longitudinally (not including the ends). For example, $m=1$ is the rigid dipole mode, where the bunches move rigidly as they execute synchrotron oscillations, $m=2$ is the quadrupole mode where the bunch head and tail oscillate longitudinally $180^{\circ}$ out of phase. Actually, this has been a simplified description of the modes of perturbation inside a bunch. The full description involves another eigen-number in the radial direction.

The $\mu$-th coupled mode will be driven if the driving narrow resonance falls on the frequency $\left(k M_{s}+\mu+m \nu_{s}\right) f_{0}$, where $f_{0}$ is the revolution frequency and $k$ is an integer. For an excitation of the $\mu$ th coupled mode and the $m$ th azimuthal mode, the growth rate derived by Sacherer is [1]

$$
\frac{1}{\tau_{m \mu}}=\frac{e \eta M I_{b} R_{s} f_{0}}{2 \pi E \nu_{s} B_{0}} D F_{m}(\Delta \phi),
$$

where $M$ is the number of bunches, ${ }^{\dagger} B_{0}=\tau_{L} f_{0}$ is the single-bunch bunching factor with $\tau_{L}$ being the total bunch length, $\nu_{s}$ is the perturbed synchrotron tune, $R_{s}$ is the shunt impedance of the sharp driving resonance at frequency $f_{r}=\omega_{r} /(2 \pi)$. The factor $D$ is a function of the decay decrement $\alpha \tau_{\text {sep }}$ between successive bunches, where $\alpha=\omega_{r} /(2 Q)$ is the half-width-athalf-maximum (HWHM) of the resonance of quality factor $Q$ and $\tau_{\text {sep }}$ is the bunch separation. It is defined as

$$
D\left(\alpha \tau_{\text {sep }}\right)=-i 2 \alpha \tau_{\text {sep }} \sum_{k=0}^{\infty} e^{-2 \pi i k \mu / M-k(\alpha-i \Omega) \tau_{\text {sep }}} \sin k \omega_{r} \tau_{\text {sep }}
$$

\footnotetext{
${ }^{\dagger}$ For a symmetrically filled ring with $M_{s}$ bunches spaced $n$-buckets apart, the number of coupled modes is $M_{s}$, which is also equal to $h / n$, where $h$ is the rf harmonic. We assume that the problem will not be significantly changed if a small fraction of bunches are missing. We therefore can have number of bunches $M \lesssim M_{s}$. In the 36 -by-36 scenario, $n=21$ so that $M_{s}=53$, but $M=36$.
} 


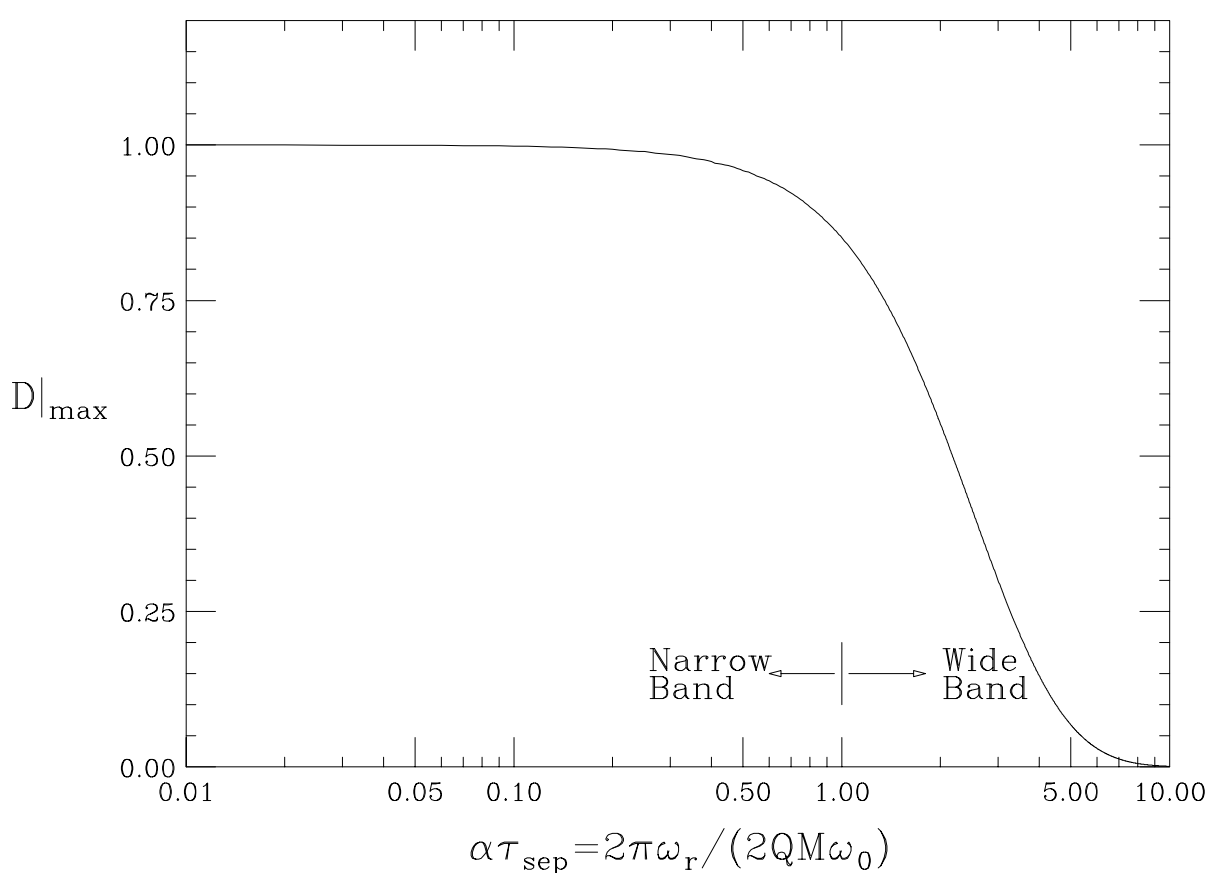

Figure 1: $|D|_{\max }$ as a function of bunch-to-bunch decay decrement $\alpha \tau_{\text {sep }}$. Note that $|D|_{\max } \approx 1$ for narrow resonances but drops very rapidly as the resonance becomes broader.

The maximum magnitude of $D$ is shown in Fig. 1. The form factor for parabolic bunches is given by

$$
F_{m}(\Delta \phi)=\frac{16 m}{\Delta \phi}\left[J_{m}^{2}\left(\frac{1}{2} \Delta \phi\right)-J_{m+1}\left(\frac{1}{2} \Delta \phi\right) J_{m-1}\left(\frac{1}{2} \Delta \phi\right)\right],
$$

where $\Delta \phi=2 \pi f_{r} \tau_{L}$ is the phase change of the resonator during the bunch passage from head to tail, and is plotted in Fig. 2. We see that mode $m$ peaks roughly at $\Delta \phi=m \pi$. This is reasonable because, as was mentioned above, mode $m$ represents a longitudinal variation along the bunch with $m$ nodes (not including the ends) and it will be most easily excited when the bunch sees a phase variation of $m \pi$ of the driving resonance as it passes through the cavity gap from head to tail. Note that $F_{m}$ decreases as $m$ increases, implying that the higher $m$ modes will not be excited so easily. 


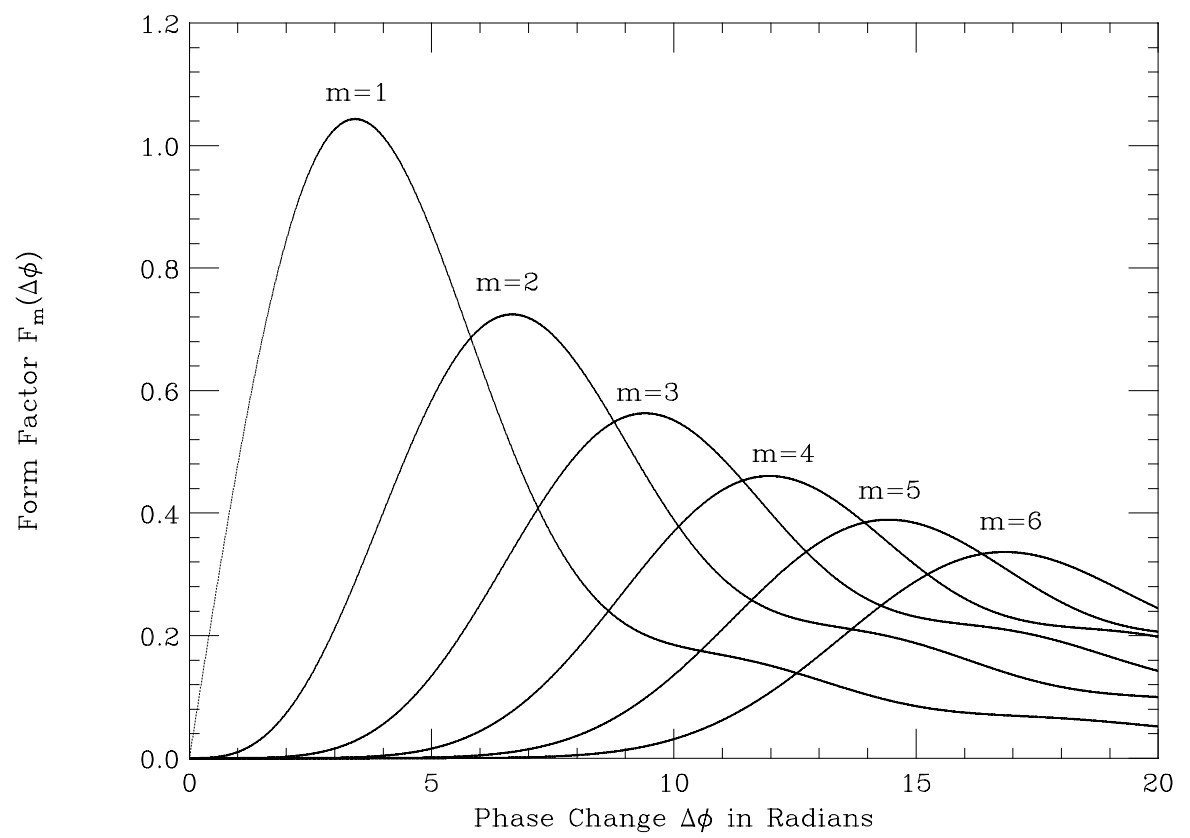

Figure 2: Form factor for longitudinal oscillation inside a bunch with $m=$ $1,2,3,4,5$ and 6 nodes.

\subsection{THE $36 \times 36$ SCENARIO OF THE TEVATRON}

The higher-order parasitic modes in the Tevatron rf cavities were measured by Sun [2] in 1995 using the method of dielectric bead pull. They were also computed using URMEL [3]. The results are listed side by side in Table I. We find that the URMEL resonant frequencies and $R / Q$ for these modes agree rather well with Sun's measurement. On the other hand, the quality factors $Q$ do not agree so well. This may be because URMEL computes the modes of the bare cavity, while some of these modes have actually been de-Qued passively. There are also a lot of structures inside the cavity and these structures have not been included in the simplified model of the cavity used in URMEL computation. In the discussions below, Sun's results will be used.

In the 36-by-36 scenario, the bunch spacing is $21 \mathrm{rf}$-buckets. If the ring is symmetrically filled, there will be $M_{s}=h / 21=53$ coupled modes, where $h=1113$ is the rf harmonic. The impedance of these higher-order parasitic resonances for one cavity is plotted in the lower trace in Fig. 3 as a function of the coupled-bunch mode $\mu$ which they will drive. The fundamental rf resonance is not included. Most of the time, the higher-order resonances of the 8 cavities will not peak at exactly the same frequencies. In this analysis, we further assume that the 
Table I: Longitudinal modes for one whole cavity.

\begin{tabular}{lrrrrrr}
\hline & \multicolumn{3}{c}{ URMEL Results } & \multicolumn{3}{c}{ Sun's Measurements } \\
Mode Type Frequency & \multicolumn{1}{c}{$R / Q$} & $Q$ & Frequency & $R / Q$ & $Q$ \\
& $(\mathrm{MHz})$ & \multicolumn{1}{c}{$(\Omega)$} & & $(\mathrm{MHz})$ & $(\Omega)$ & \\
\hline TM0-EE-1 & 53.49 & 87.65 & 9537 & 53.11 & 109.60 & 6523 \\
TM0-ME-1 & 84.10 & 22.61 & 12819 & 56.51 & 18.81 & 3620 \\
TM0-EE-2 & 166.56 & 18.47 & 16250 & 158.23 & 11.68 & 6060 \\
TM0-ME-2 & 188.94 & 10.83 & 18235 & & & \\
TM0-EE-3 & 285.94 & 7.53 & 20524 & 310.68 & 7.97 & 15923 \\
TM0-ME-3 & 308.46 & 4.07 & 22660 & & & \\
TM0-EE-4 & 402.69 & 4.93 & 25486 & 439.77 & 5.23 & 13728 \\
TM0-ME-4 & 431.34 & 1.72 & 26407 & 424.25 & 1.28 & 6394 \\
TM0-EE-5 & 511.69 & 5.57 & 25486 & 559.48 & 6.73 & 13928 \\
TM0-ME-5 & 549.57 & 1.36 & 29453 & & & \\
& & & & 748.18 & 10.90 & 13356 \\
& & & & 768.03 & 2.47 & 16191 \\
\hline
\end{tabular}

resonances of the 8 cavities overlap each other in such a way that the total shunt impedance remains the same as for one resonance while the total width becomes broadened (or de-Qued) 8 times. $^{\ddagger}$ The driving impedance of all the 8 cavities are shown in the upper trace in Fig. 3 . Although de-Qued, each resonance is still narrow enough to maintain a value of $|D|>0.95$ in Eq. (1.2) if its peak falls on a synchrotron sideband. However, the resonances are broad enough to cover a number of revolution harmonics. As a result, nearly every coupled-bunch mode is affected. Above transition, the upper synchrotron sidebands correspond to growth while the lower synchrotron sidebands correspond to damping. As a result, some coupled-bunch modes will grow and some will be damped.

The growth rate for each coupled-bunch mode is computed at the injection energy of $150 \mathrm{GeV}$ for each higher-order parasitic resonance. The total growth rate is obtained by summing the contribution from all the resonances. The results are listed in Table II. The injection energy was chosen because the growth rate is inversely proportional to energy and therefore the most severe instabilities will occur at the injection energy. These growth rates

\footnotetext{
${ }^{\ddagger}$ If the total width is broadened only $n<8$ times, the total shunt impedance will be increased $8 / n$ times. The total number of coupled-bunch modes driven will be less; but the growth or damping rates will be faster.
} 


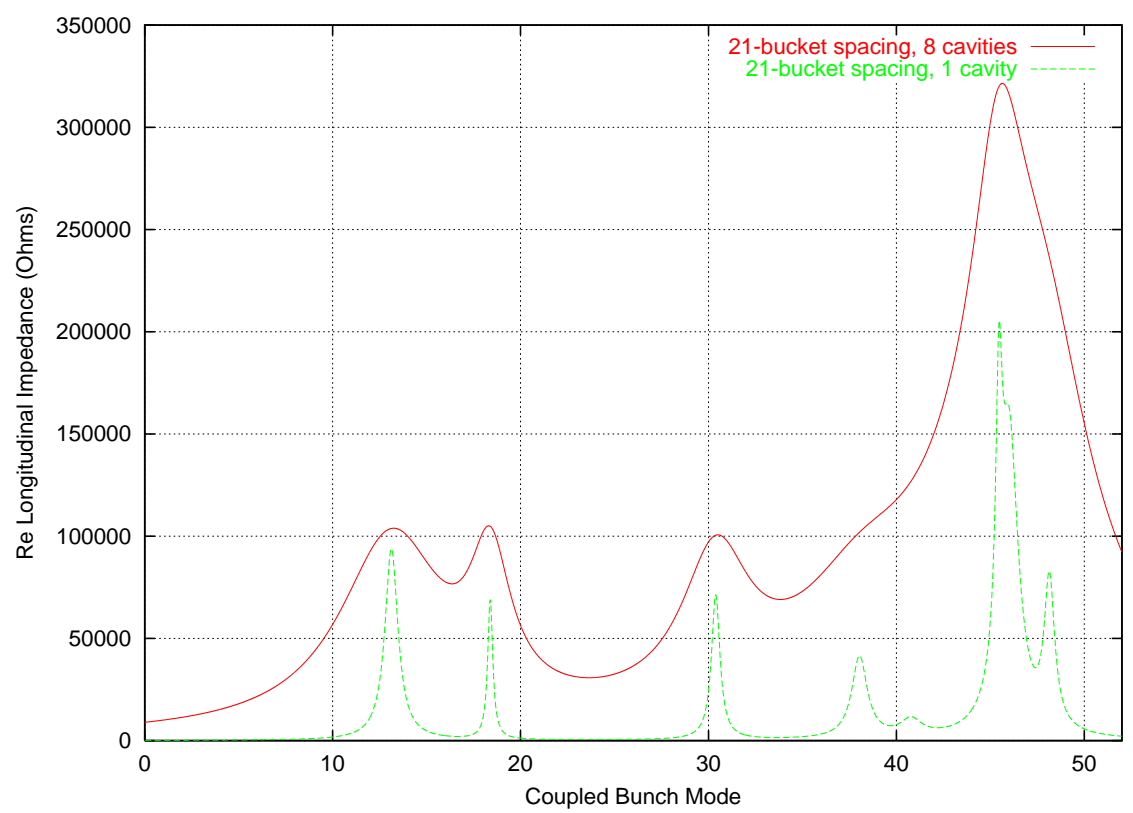

Figure 3: (color) The real part of the impedance of the higher-order parasitic resonances are plotted as a function of the coupled-bunch mode $\mu$ that they will drive. The lower (green) trace is for one cavity while the upper (red) trace corresponds to 8 cavities assuming that each resonance spread out 8 folds. The bunch spacing is 21 buckets.

and damping rates are also plotted in Fig. 4. A resonance that drives coupled-bunch mode $\mu$ will also damp coupled-bunch mode $M_{s}-\mu$. Thus a resonance at $\mu$ and one at $M_{s}-\mu$ will compensate each other for both coupled-bunch modes $\mu$ and $M_{s}-\mu$. Unfortunately, we do not have such matching resonance pairs in Fig. 3. As a result, as indicated in Fig. 4, the coupled modes that grow are not compensated very much by other resonances.

There is a spread of the synchrotron frequency due to the nonlinear sinusoidal rf wave form. This spread from the center to the edge of the bunch is given by

$$
\frac{\Delta \omega_{s}}{\omega_{s}}=\frac{1}{16}\left(\frac{1+\Gamma^{2}}{1-\Gamma^{2}}\right)\left(\frac{h \tau_{L} f_{0}}{2}\right)^{2}=0.00353 \text { or } \Delta f_{s}=0.308 \mathrm{~Hz},
$$

where the rms bunch length $\tau_{L}$ has been taken as $60 \mathrm{~cm}$, the nominal synchrotron tune $\nu_{s}=1.83 \times 10^{-3}$ is assumed at the injection energy of $150 \mathrm{GeV}$ with an rf voltage of $1 \mathrm{MV}$ and the synchronous phase $\phi_{s}=\sin ^{-1} \Gamma$ is taken to be zero. This spread supplies Landau damping. The mode will be stable if

$$
\frac{1}{\tau}<\frac{\sqrt{m}}{4} \Delta \omega_{s}=0.483 \sqrt{m} \mathrm{~s}^{-1}
$$

The Landau damping rates are listed in the last row of Table V. We see that all coupled modes 
Table II: Longitudinal coupled-bunch growth rates driven by the higher-order modes of the rf cavities at injection for the $36 \times 36$ scenario in Run II with rms bunch length $60 \mathrm{~cm}$ and bunch intensity $1.7 \times 10^{11}$.

\begin{tabular}{|c|c|c|c|c|c|c|}
\hline \multirow{2}{*}{$\begin{array}{r}\text { Coupled } \\
\text { Bunch Mode }\end{array}$} & \multicolumn{6}{|c|}{ Growth Rate in $\mathrm{sec}^{-1}$} \\
\hline & $m=1$ & $m=2$ & $m=3$ & $m=4$ & $m=5$ & $m=6$ \\
\hline 0 & -0.549 & -0.085 & 0.010 & -0.042 & 0.010 & -0.043 \\
\hline 1 & -0.059 & -0.102 & -0.171 & -0.174 & -0.133 & -0.141 \\
\hline 2 & -0.107 & -0.195 & -0.272 & -0.247 & -0.177 & -0.178 \\
\hline 3 & -0.148 & -0.270 & -0.374 & -0.336 & -0.234 & -0.225 \\
\hline 4 & -0.202 & -0.370 & -0.500 & -0.439 & -0.299 & -0.280 \\
\hline 5 & -0.260 & -0.484 & -0.613 & -0.514 & -0.347 & -0.330 \\
\hline 6 & -0.332 & -0.636 & -0.710 & -0.536 & -0.357 & -0.361 \\
\hline 7 & -0.435 & -0.857 & -0.832 & -0.537 & -0.339 & -0.362 \\
\hline 8 & -0.417 & -0.827 & -0.770 & -0.463 & -0.285 & -0.330 \\
\hline 9 & -0.265 & -0.517 & -0.507 & -0.314 & -0.200 & -0.270 \\
\hline 10 & -0.153 & -0.292 & -0.308 & -0.182 & -0.107 & -0.198 \\
\hline 11 & -0.084 & -0.154 & -0.174 & -0.069 & -0.013 & -0.125 \\
\hline 12 & -0.040 & -0.065 & -0.082 & 0.021 & 0.067 & -0.068 \\
\hline 13 & -0.013 & -0.012 & -0.025 & 0.076 & 0.115 & -0.031 \\
\hline 14 & 0.026 & 0.004 & -0.011 & 0.076 & 0.109 & -0.029 \\
\hline 15 & 0.010 & 0.010 & -0.003 & 0.051 & 0.072 & -0.047 \\
\hline 16 & 0.032 & -0.002 & -0.005 & 0.032 & 0.041 & -0.057 \\
\hline 17 & 0.098 & -0.018 & -0.018 & 0.009 & 0.016 & -0.060 \\
\hline 18 & 0.235 & -0.029 & -0.022 & -0.001 & 0.004 & -0.055 \\
\hline 19 & 0.143 & -0.054 & -0.025 & -0.006 & -0.002 & -0.048 \\
\hline 20 & -0.107 & -0.096 & -0.029 & -0.008 & -0.005 & -0.042 \\
\hline 21 & -0.327 & -0.157 & -0.035 & -0.009 & -0.006 & -0.036 \\
\hline 22 & -0.492 & -0.210 & -0.041 & -0.009 & -0.006 & -0.032 \\
\hline 23 & -0.552 & -0.217 & -0.040 & -0.009 & -0.006 & -0.028 \\
\hline 24 & -0.377 & -0.147 & -0.025 & -0.002 & -0.001 & -0.018 \\
\hline 25 & -0.195 & -0.075 & -0.013 & 0.000 & 0.001 & -0.014 \\
\hline 26 & -0.059 & -0.024 & -0.005 & -0.002 & -0.001 & -0.002 \\
\hline 27 & 0.060 & 0.024 & 0.005 & 0.002 & 0.001 & 0.002 \\
\hline 28 & 0.195 & 0.075 & 0.013 & 0.000 & -0.001 & 0.014 \\
\hline 29 & 0.377 & 0.147 & 0.025 & 0.002 & 0.001 & 0.018 \\
\hline 30 & 0.552 & 0.218 & 0.040 & 0.009 & 0.006 & 0.028 \\
\hline 31 & 0.491 & 0.210 & 0.041 & 0.009 & 0.006 & 0.032 \\
\hline 32 & 0.326 & 0.156 & 0.035 & 0.009 & 0.006 & 0.036 \\
\hline 33 & 0.106 & 0.096 & 0.029 & 0.008 & 0.004 & 0.042 \\
\hline 34 & -0.144 & 0.053 & 0.025 & 0.006 & 0.002 & 0.048 \\
\hline
\end{tabular}


Table II continued.

\begin{tabular}{|c|c|c|c|c|c|c|}
\hline \multirow{2}{*}{$\begin{array}{r}\text { Coupled } \\
\text { Bunch Mode }\end{array}$} & \multicolumn{6}{|c|}{ Growth Rate in $\mathrm{sec}^{-1}$} \\
\hline & $m=1$ & $m=2$ & $m=3$ & $m=4$ & $m=5$ & $m=6$ \\
\hline 35 & -0.235 & 0.029 & 0.022 & 0.000 & -0.005 & 0.055 \\
\hline 36 & -0.097 & 0.018 & 0.018 & -0.009 & -0.017 & 0.060 \\
\hline 37 & -0.032 & 0.002 & 0.005 & -0.032 & -0.041 & 0.057 \\
\hline 38 & -0.010 & -0.010 & 0.002 & -0.052 & -0.072 & 0.046 \\
\hline 39 & -0.026 & -0.004 & 0.011 & -0.077 & -0.110 & 0.029 \\
\hline 40 & 0.013 & 0.013 & 0.025 & -0.076 & -0.115 & 0.031 \\
\hline 41 & 0.040 & 0.066 & 0.083 & -0.020 & -0.066 & 0.069 \\
\hline 42 & 0.084 & 0.155 & 0.175 & 0.070 & 0.014 & 0.126 \\
\hline 43 & 0.153 & 0.293 & 0.310 & 0.184 & 0.109 & 0.200 \\
\hline 44 & 0.265 & 0.520 & 0.510 & 0.316 & 0.201 & 0.272 \\
\hline 45 & 0.418 & 0.829 & 0.773 & 0.465 & 0.286 & 0.331 \\
\hline 46 & 0.434 & 0.856 & 0.831 & 0.537 & 0.339 & 0.362 \\
\hline 47 & 0.332 & 0.635 & 0.708 & 0.536 & 0.358 & 0.360 \\
\hline 48 & 0.260 & 0.483 & 0.612 & 0.513 & 0.346 & 0.329 \\
\hline 49 & 0.201 & 0.369 & 0.499 & 0.438 & 0.298 & 0.278 \\
\hline 50 & 0.148 & 0.269 & 0.373 & 0.334 & 0.233 & 0.224 \\
\hline 51 & 0.107 & 0.194 & 0.271 & 0.246 & 0.176 & 0.177 \\
\hline 52 & 0.059 & 0.102 & 0.170 & 0.173 & 0.133 & 0.140 \\
\hline \multicolumn{7}{|c|}{ Laudau Damping rate $\left(\mathrm{s}^{-1}\right)$} \\
\hline & 0.483 & 0.684 & 0.837 & 0.967 & 1.081 & 1.184 \\
\hline
\end{tabular}

are damped with the exception of the dipole mode $(m=1)$ in coupled modes $\mu=30$ and 31 , and the quadrupole mode $(m=2)$ in the coupled modes $\mu=45,46$, and 47 .

We would like to point out that the stability criterion mentioned in Eq. (1.5) is a rough Keil-Schnell type criterion. The actual stability criterion is distribution dependent and is also nonsymmetric with respect to the sign of the reactive impedance. As an example, consider the inductive impedance of the vacuum chamber, which gives rise to an incoherent synchrotron frequency shift of

$$
\frac{\Delta \omega_{s}}{\omega_{s}}=-\frac{3 I_{b} \mathcal{I} m\left(Z_{\|} / n\right)}{2 \pi^{2} h V_{\mathrm{rf}} \cos \phi_{s} B_{0}^{3}}=-0.00684 \quad \text { or } \quad \Delta f_{s}=-0.595 \mathrm{~Hz},
$$

where $\operatorname{Im}\left(Z_{\|} / n\right)=3 \Omega$ has been used. This shift is towards lower frequencies because the Tevatron is operated above transition. However, the coherent synchrotron frequency remains the same as the unperturbed synchrotron frequency $f_{s 0}$. Thus the incoherent spread of the 


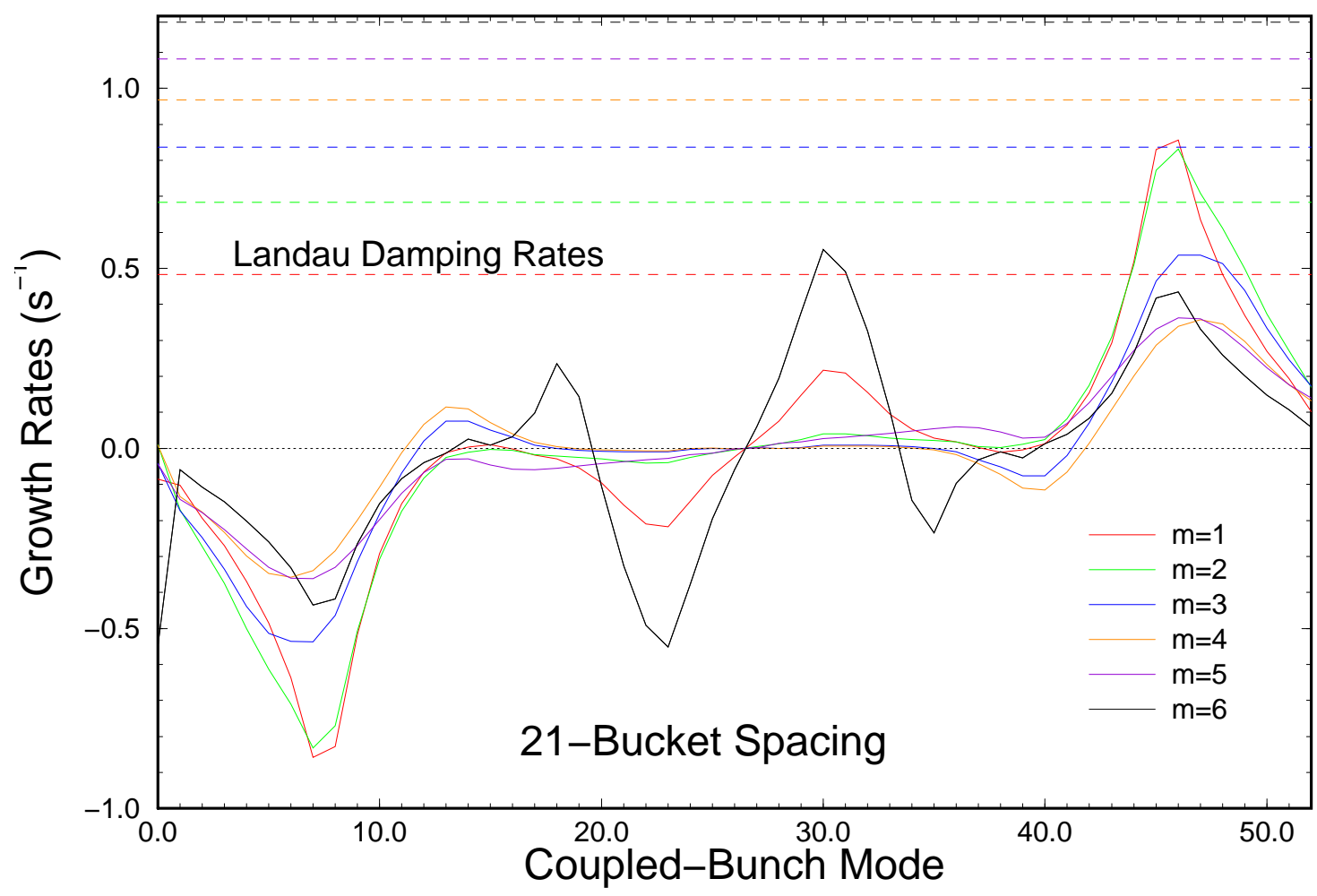

Figure 4: (color) Plot of growth rates (positive) and damping rates (negative) of each coupled-bunch mode driven by the higher-order parasitic resonances of the 8 Tevatron cavities for the 36-by-36 scenario. Landau damping rates are shown in dashes.

synchrotron frequency will not cover $f_{s 0}$, and will not supply any damping at all. This is illustrated in Fig. 5. However, when the impedance involves a real part, it can be shown that Landau damping may exist if the real part is not too large. On the other hand, if we consider an unperturbed distribution with a vanishing gradient at the edge, the situation will be different and some Landau damping does exist even if the impedance is purely inductive. In short, more detailed solution of a dispersion relation is necessary to determine whether a certain mode is damped or antidamped.

If the growth turns out to be harmful, a fast $36 \times 36$ bunch-by-bunch damper may be necessary to damp the dipole mode $(m=1)$. A bunch-to-bunch damper for the quadrupole mode $(m=2)$ may also be necessary. This consists essentially of a wall-gap pickup monitoring the changes in bunch length and the corresponding excitation of a modulation of the rf waveform with roughly twice the synchrotron frequency.

The Tevatron bunches will be formed by coalescing 9 or more bunches in the Main Injector 


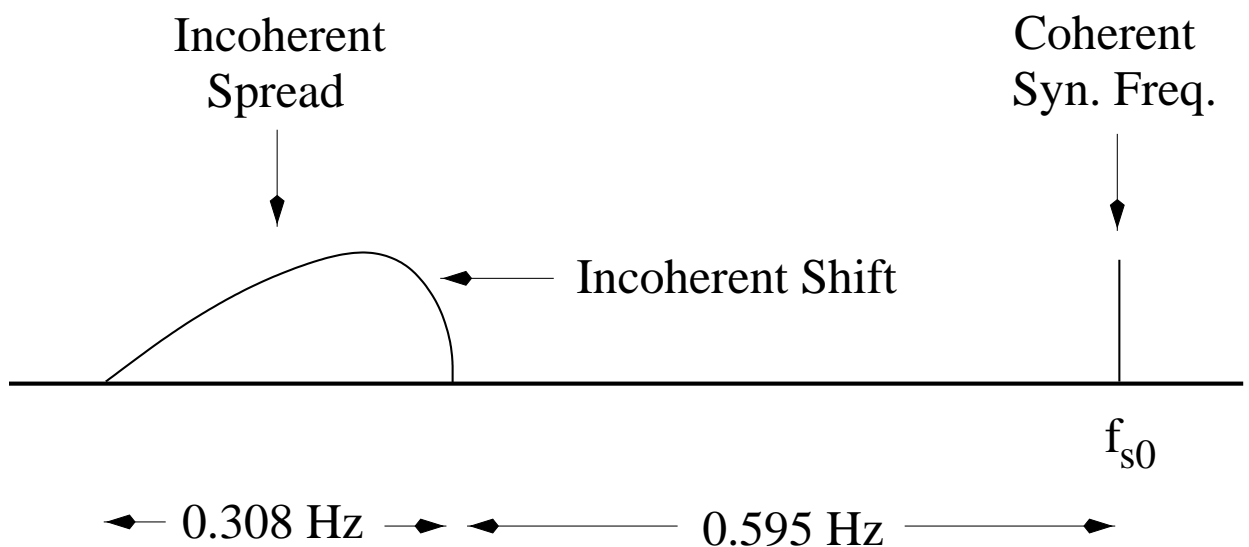

Figure 5: Schematic drawing showing the incoherent spread of $\Delta f_{s} \approx 0.308 \mathrm{~Hz}$ is shifted by $-0.595 \mathrm{~Hz}$ from the coherent synchrotron frequency $f_{s 0}$, thus not being able to provide Landau damping.

(formerly in the Main Ring). Usually there will be a $10 \%$ difference in the number of particles in the final bunches. Each bunch will be experiencing a slightly different wake force and will therefore be driven slightly differently. Such broken symmetry of the coupled-bunch system will lead to some damping also.

\subsection{THE $108 \times 108$ SCENARIO}

We would like also to compute the longitudinal coupled-bunch growth rates for the 108-by108 scenario. The 108 bunches are mostly at 7 -bucket spacing and contain $2.7 \times 10^{11}$ particles each. The rms bunch length is expected to be $50 \mathrm{~cm}$. If the ring is symmetrically filled with 7-bucket spacing, there will be in total $M_{s}=h / 7=159$ coupled-bunch modes. For each coupled-bunch mode, the real part of the impedance of the higher-order parasitic resonances in the rf cavities driving the coupled-bunch mode $\mu=0,1,2, \cdots, 158$ are plotted in Fig. 6. The lower trace is for one cavity. The upper trace is for all 8 cavities with the assumption that the quality factors will be de-Qued 8 folds due to the fact that these resonances will not peak at exactly the same frequencies in the 8 cavities. Here, the resonances appear not as crowded as in the 21-bucket spacing in Fig. 3, the reason being that there are 3 times as many coupled modes here. We also notice that the resonances do not come in complementary pairs, i.e., $\mu$ and $M_{s}-\mu$. As a result, the resonances are not helping each other much in growth reduction. The growth rates at $150 \mathrm{GeV}$ are computed as in the $36 \times 36$ scenario. The results are plotted in Fig. 7 for each of the 159 coupled-bunch modes. They are also listed in Table III with the 


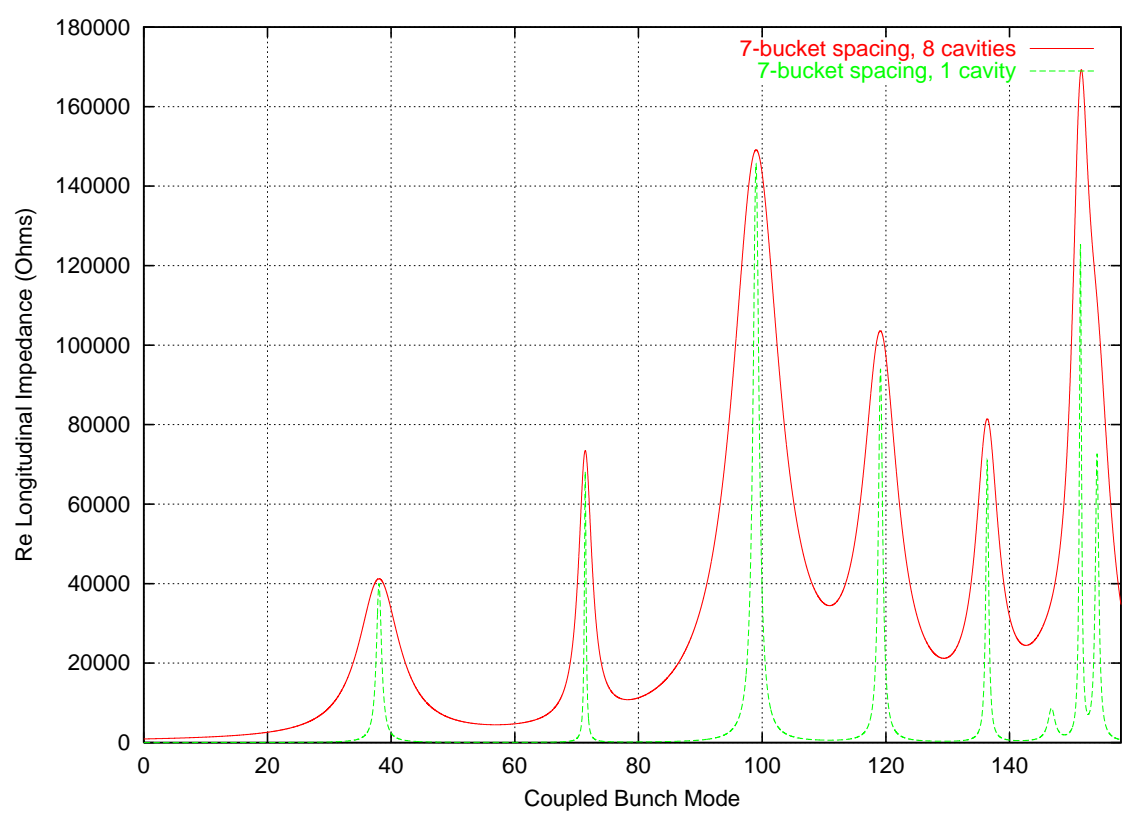

Figure 6: (color) The real part of the impedance of the higher-order parasitic resonances are plotted as a function of the coupled-bunch mode $\mu$ that they will drive. The bunches are at 7-bucket spacing. The lower (green) trace is for one cavity while the upper (red) trace corresponds to 8 cavities assuming that each resonance spread out 8 folds.

rates of Landau damping due to synchrotron frequency spread in the last row.

We see that with the number of bunches tripled and the bunch intensity increased by about $60 \%$, coupled-bunch growth rates become very much faster. It appears that the spread in synchrotron frequency is not large enough to provide the necessary Landau damping. Obviously a fast bunch-to-bunch damper is required for the dipole mode $(m=1)$ and the quadrupole mode $(m=2)$. Some higher azimuthal modes $(m>2)$ are also unstable and cannot be damped by bunch-by-bunch dampers. To stabilize these modes and to reduce the growth rates of the dipole and quadrupole modes, one may resort to (1) passively de-Quing some annoying parasitic resonances inside the cavities and (2) increasing the bunch length. A longer bunch length will push the excitation to higher azimuthal modes, whose growth rates are usually slower and the corresponding Landau damping larger. However, longer bunches implies lower luminosity. This is especially true in the $108 \times 108$ scenario, where the bunches collide at a small angle. 
Table III: Longitudinal coupled-bunch growth rates driven by the higher-order modes of the rf cavities at injection for the $108 \times 108$ scenario in Run II with rms bunch length $60 \mathrm{~cm}$ and bunch intensity $2.7 \times 10^{11}$.

\begin{tabular}{rrrrrrr}
\hline Coupled & & \multicolumn{5}{c}{ Growth Rate in sec } \\
Bunch Mode & $m=1$ & $m=2$ & $m=3$ & $m=4$ & $m=5$ & $m=6$ \\
\hline 0 & -2.676 & -0.257 & 0.049 & -0.023 & -0.595 & -0.638 \\
1 & -0.257 & -0.603 & -0.690 & -0.363 & -0.106 & -0.020 \\
2 & -0.625 & -1.208 & -1.135 & -0.549 & -0.155 & -0.029 \\
3 & -0.894 & -1.726 & -1.622 & -0.785 & -0.222 & -0.041 \\
4 & -1.267 & -2.403 & -2.208 & -1.057 & -0.298 & -0.055 \\
5 & -1.720 & -3.085 & -2.624 & -1.203 & -0.332 & -0.060 \\
6 & -2.386 & -3.885 & -2.801 & -1.151 & -0.300 & -0.053 \\
7 & -3.431 & -5.133 & -3.074 & -1.067 & -0.249 & -0.041 \\
8 & -3.385 & -4.937 & -2.768 & -0.890 & -0.195 & -0.031 \\
9 & -2.099 & -3.117 & -1.827 & -0.617 & -0.141 & -0.023 \\
10 & -1.235 & -1.901 & -1.208 & -0.441 & -0.106 & -0.018 \\
11 & -0.800 & -1.284 & -0.882 & -0.344 & -0.086 & -0.015 \\
12 & -0.568 & -0.948 & -0.696 & -0.284 & -0.072 & -0.012 \\
13 & -0.430 & -0.741 & -0.571 & -0.240 & -0.062 & -0.011 \\
14 & -0.337 & -0.596 & -0.475 & -0.204 & -0.053 & -0.009 \\
15 & -0.397 & -0.356 & -0.314 & -0.153 & -0.043 & -0.008 \\
16 & -0.419 & -0.239 & -0.161 & -0.073 & -0.019 & -0.003 \\
17 & -0.530 & -0.247 & -0.139 & -0.061 & -0.016 & -0.003 \\
18 & -0.708 & -0.275 & -0.123 & -0.052 & -0.014 & -0.002 \\
19 & -1.008 & -0.337 & -0.113 & -0.044 & -0.011 & -0.002 \\
20 & -1.522 & -0.457 & -0.111 & -0.037 & -0.010 & -0.002 \\
21 & -2.370 & -0.666 & -0.119 & -0.033 & -0.008 & -0.001 \\
22 & -3.377 & -0.917 & -0.134 & -0.030 & -0.007 & -0.001 \\
23 & -3.530 & -0.949 & -0.130 & -0.026 & -0.006 & -0.001 \\
24 & -2.590 & -0.680 & -0.070 & 0.015 & 0.045 & 0.047 \\
25 & -1.657 & -0.432 & -0.038 & 0.024 & 0.053 & 0.054 \\
26 & -1.065 & -0.273 & -0.014 & 0.034 & 0.063 & 0.063 \\
27 & -0.716 & -0.176 & 0.005 & 0.044 & 0.075 & 0.074 \\
28 & -0.557 & -0.199 & -0.124 & -0.101 & -0.003 & 0.055 \\
29 & -0.429 & -0.169 & -0.132 & -0.114 & 0.000 & 0.066 \\
30 & -0.347 & -0.153 & -0.146 & -0.131 & 0.003 & 0.081 \\
31 & -0.294 & -0.147 & -0.166 & -0.153 & 0.007 & 0.101 \\
32 & -0.074 & -0.100 & -0.189 & -0.182 & 0.013 & 0.128 \\
& -0.089 & -0.119 & -0.229 & -0.222 & 0.021 & 0.164 \\
& -0.112 & -0.146 & -0.287 & -0.281 & 0.029 & 0.211 \\
\hline 34
\end{tabular}


Table III continued.

\begin{tabular}{|c|c|c|c|c|c|c|}
\hline \multirow{2}{*}{$\begin{array}{r}\text { Coupled } \\
\text { Bunch Mode }\end{array}$} & \multicolumn{6}{|c|}{ Growth Rate in $\mathrm{sec}^{-1}$} \\
\hline & $m=1$ & $m=2$ & $m=3$ & $m=4$ & $m=5$ & $m=6$ \\
\hline 35 & -0.146 & -0.189 & -0.374 & -0.371 & 0.030 & 0.268 \\
\hline 36 & -0.195 & -0.252 & -0.504 & -0.511 & 0.008 & 0.325 \\
\hline 37 & -0.280 & -0.379 & -0.732 & -0.752 & -0.077 & 0.353 \\
\hline 38 & -0.404 & -0.574 & -1.068 & -1.112 & -0.262 & 0.313 \\
\hline 39 & -0.537 & -0.793 & -1.430 & -1.505 & -0.513 & 0.202 \\
\hline 40 & -0.601 & -0.909 & -1.610 & -1.704 & -0.682 & 0.086 \\
\hline 41 & -0.553 & -0.843 & -1.484 & -1.574 & -0.668 & 0.026 \\
\hline 42 & -0.476 & -0.751 & -1.284 & -1.380 & -0.702 & -0.130 \\
\hline 43 & -0.368 & -0.588 & -0.995 & -1.074 & -0.577 & -0.141 \\
\hline 44 & -0.286 & -0.465 & -0.776 & -0.844 & -0.487 & -0.154 \\
\hline 45 & -0.230 & -0.382 & -0.626 & -0.687 & -0.434 & -0.174 \\
\hline 46 & -0.194 & -0.330 & -0.529 & -0.588 & -0.412 & -0.201 \\
\hline 47 & -0.171 & -0.301 & -0.470 & -0.529 & -0.412 & -0.235 \\
\hline 48 & -0.159 & -0.289 & -0.439 & -0.501 & -0.431 & -0.276 \\
\hline 49 & -0.154 & -0.291 & -0.429 & -0.498 & -0.468 & -0.327 \\
\hline 50 & -0.157 & -0.305 & -0.439 & -0.516 & -0.524 & -0.389 \\
\hline 51 & -0.166 & -0.332 & -0.467 & -0.555 & -0.601 & -0.467 \\
\hline 52 & -0.182 & -0.372 & -0.514 & -0.618 & -0.703 & -0.565 \\
\hline 53 & -0.171 & -0.378 & -0.492 & -0.611 & -0.807 & -0.705 \\
\hline 54 & -0.209 & -0.463 & -0.602 & -0.749 & -0.988 & -0.863 \\
\hline 55 & -0.258 & -0.572 & -0.743 & -0.924 & -1.219 & -1.065 \\
\hline 56 & -0.319 & -0.707 & -0.918 & -1.143 & -1.507 & -1.317 \\
\hline 57 & -0.391 & -0.866 & -1.125 & -1.400 & -1 & -1.613 \\
\hline 58 & -0.466 & -1.033 & -1.341 & -1.668 & -2.201 & -1.922 \\
\hline 59 & -0.526 & -1.167 & -1.515 & -1.885 & -2.486 & -2.170 \\
\hline 60 & -0.550 & -1.220 & -1.583 & -1.969 & -2.597 & -2.266 \\
\hline 61 & -0.526 & -1.166 & -1.513 & -1.883 & -2.482 & -2.165 \\
\hline 62 & -0.466 & -1.032 & -1.339 & -1.665 & -2.195 & -1.914 \\
\hline 63 & -0.391 & -0.866 & -1.123 & -1.397 & -1.841 & -1.605 \\
\hline 64 & -0.319 & -0.707 & -0.916 & -1.140 & -1.503 & -1.309 \\
\hline 65 & -0.258 & -0.572 & -0.741 & -0.922 & -1.215 & -1.059 \\
\hline 66 & -0.209 & -0.463 & -0.601 & -0.748 & -0.985 & -0.858 \\
\hline 67 & -0.019 & -0.374 & -0.491 & -0.611 & -0.805 & -0.701 \\
\hline 68 & 0.101 & -0.306 & -0.405 & -0.505 & -0.665 & -0.579 \\
\hline 69 & 0.314 & -0.248 & -0.338 & -0.422 & -0.555 & -0.483 \\
\hline 70 & 0.781 & -0.194 & -0.286 & -0.356 & -0.469 & -0.408 \\
\hline 71 & 1.633 & -0.137 & -0.243 & -0.304 & -0.400 & -0.348 \\
\hline 72 & 1.454 & -0.117 & -0.210 & -0.262 & -0.345 & -0.300 \\
\hline
\end{tabular}


Table III continued.

\begin{tabular}{|c|c|c|c|c|c|c|}
\hline \multirow{2}{*}{$\begin{array}{r}\text { Coupled } \\
\text { Bunch Mode }\end{array}$} & \multicolumn{6}{|c|}{ Growth Rate in $\sec ^{-1}$} \\
\hline & $m=1$ & $m=2$ & $m=3$ & $m=4$ & $m=5$ & $m=6$ \\
\hline 73 & 0.676 & -0.119 & -0.182 & -0.228 & -0.300 & -0.261 \\
\hline 74 & 0.318 & -0.112 & -0.160 & -0.199 & -0.263 & -0.228 \\
\hline 75 & 0.167 & -0.103 & -0.141 & -0.176 & -0.232 & -0.202 \\
\hline 76 & -0.044 & -0.097 & -0.126 & -0.157 & -0.206 & -0.179 \\
\hline 77 & -0.039 & -0.087 & -0.112 & -0.140 & -0.184 & -0.160 \\
\hline 77 & -0.039 & -0.087 & -0.112 & -0.140 & -0.184 & -0.160 \\
\hline 78 & -0.035 & -0.078 & -0.101 & -0.126 & -0.166 & -0.144 \\
\hline 79 & 0.000 & 0.000 & 0.000 & 0.000 & 0.000 & 0.000 \\
\hline 80 & 0.000 & 0.000 & 0.000 & 0.000 & 0.000 & 0.000 \\
\hline 81 & 0.035 & 0.078 & 0.101 & 0.126 & 0.166 & 0.144 \\
\hline 82 & 0.039 & 0.087 & 0.112 & 0.140 & 0.185 & 0.161 \\
\hline 83 & 0.044 & 0.097 & 0.126 & 0.157 & 0.207 & 0.180 \\
\hline 84 & -0.168 & 0.103 & 0.141 & 0.176 & 0.232 & 0.202 \\
\hline 85 & -0.319 & 0.112 & 0.160 & 0.200 & 0.263 & 0.229 \\
\hline 86 & -0.678 & 0.119 & 0.183 & 0.228 & 0.300 & 0.262 \\
\hline 87 & -1.458 & 0.117 & 0.210 & 0.262 & 0.346 & 0.301 \\
\hline 88 & -1.630 & 0.138 & 0.244 & 0.305 & 0.401 & 0.350 \\
\hline 89 & -0.778 & 0.195 & 0.286 & 0.357 & 0.470 & 0.410 \\
\hline 90 & -0.313 & 0.249 & 0.339 & 0.423 & 0.557 & 0.485 \\
\hline 91 & -0.101 & 0.306 & 0.406 & 0.506 & 0.667 & 0.581 \\
\hline 92 & 0.019 & 0.375 & 0.492 & 0.613 & 0.808 & 0.704 \\
\hline 93 & 0.209 & 0.464 & 0.602 & 0.750 & 0.989 & 0.862 \\
\hline 94 & 0.258 & 0.572 & 0.743 & 0.925 & 1.220 & 1.064 \\
\hline 95 & 0.319 & 0.708 & 0.918 & 1.144 & 1.508 & 1.316 \\
\hline 96 & 0.391 & 0.867 & 1.125 & 1.401 & 1.847 & 1.612 \\
\hline 97 & 0.466 & 1.033 & 1.341 & 1.669 & 2.201 & 1.921 \\
\hline 98 & 0.526 & 1.167 & 1.515 & 1.885 & 2.486 & 2.169 \\
\hline 99 & 0.550 & 1.220 & 1.583 & 1.969 & 2.597 & 2.266 \\
\hline 100 & 0.526 & 1.166 & 1.513 & 1.882 & 2.482 & 2.166 \\
\hline 101 & 0.465 & 1.031 & 1.339 & 1.664 & 2.195 & 1.915 \\
\hline 102 & 0.390 & 0.865 & 1.123 & 1.396 & 1.840 & 1.606 \\
\hline 103 & 0.319 & 0.706 & 0.916 & 1.139 & 1.502 & 1.311 \\
\hline 104 & 0.258 & 0.571 & 0.741 & 0.921 & 1.214 & 1.060 \\
\hline 105 & 0.209 & 0.463 & 0.601 & 0.746 & 0.984 & 0.859 \\
\hline 106 & 0.171 & 0.378 & 0.491 & 0.610 & 0.804 & 0.702 \\
\hline 107 & 0.182 & 0.372 & 0.514 & 0.617 & 0.701 & 0.562 \\
\hline 108 & 0.166 & 0.331 & 0.466 & 0.554 & 0.599 & 0.465 \\
\hline 109 & 0.157 & 0.305 & 0.439 & 0.515 & 0.523 & 0.388 \\
\hline
\end{tabular}


Table III continued.

\begin{tabular}{|c|c|c|c|c|c|c|}
\hline \multirow{2}{*}{$\begin{array}{r}\text { Coupled } \\
\text { Bunch Mode }\end{array}$} & \multicolumn{6}{|c|}{ Growth Rate in $\mathrm{sec}^{-1}$} \\
\hline & $m=1$ & $m=2$ & $m=3$ & $m=4$ & $m=5$ & $m=6$ \\
\hline 110 & 0.154 & 0.291 & 0.429 & 0.498 & 0.468 & 0.326 \\
\hline 111 & 0.159 & 0.289 & 0.439 & 0.501 & 0.431 & 0.275 \\
\hline 112 & 0.171 & 0.302 & 0.470 & 0.530 & 0.412 & 0.234 \\
\hline 113 & 0.194 & 0.331 & 0.530 & 0.589 & 0.412 & 0.200 \\
\hline 114 & 0.230 & 0.383 & 0.627 & 0.689 & 0.435 & 0.174 \\
\hline 115 & 0.287 & 0.465 & 0.778 & 0.847 & 0.488 & 0.154 \\
\hline 116 & 0.369 & 0.589 & 0.998 & 1.078 & 0.579 & 0.140 \\
\hline 117 & 0.477 & 0.752 & 1.287 & 1.385 & 0.704 & 0.130 \\
\hline 118 & 0.554 & 0.844 & 1.486 & 1.578 & 0.670 & -0.026 \\
\hline 119 & 0.601 & 0.908 & 1.610 & 1.703 & 0.681 & -0.088 \\
\hline 120 & 0.536 & 0.792 & 1.427 & 1.500 & 0.509 & -0.205 \\
\hline 121 & 0.404 & 0.573 & 1.064 & 1.106 & 0.258 & -0.315 \\
\hline 122 & 0.280 & 0.378 & 0.729 & 0.748 & 0.074 & -0.353 \\
\hline 123 & 0.194 & 0.251 & 0.502 & 0.508 & -0.009 & -0.324 \\
\hline 124 & 0.146 & 0.189 & 0.373 & 0.369 & -0.030 & -0.266 \\
\hline 125 & 0.112 & 0.146 & 0.286 & 0.280 & -0.028 & -0.210 \\
\hline 126 & 0.089 & 0.118 & 0.228 & 0.222 & -0.021 & -0.163 \\
\hline 127 & 0.074 & 0.100 & 0.189 & 0.181 & -0.013 & -0.127 \\
\hline 128 & 0.294 & 0.147 & 0.165 & 0.152 & -0.007 & -0.101 \\
\hline 129 & 0.347 & 0.153 & 0.146 & 0.130 & -0.003 & -0.081 \\
\hline 130 & 0.429 & 0.170 & 0.132 & 0.114 & 0.000 & -0.066 \\
\hline 131 & 0.558 & 0.199 & 0.124 & 0.101 & 0.003 & -0.054 \\
\hline 132 & 0.717 & 0.176 & -0.004 & -0.044 & -0.075 & -0.073 \\
\hline 133 & 1.067 & 0.274 & 0.014 & -0.034 & -0.063 & -0.063 \\
\hline 134 & 1.659 & 0.434 & 0.038 & -0.024 & -0.053 & -0.054 \\
\hline 135 & 2.594 & 0.682 & 0.070 & -0.015 & -0.045 & -0.047 \\
\hline 136 & 3.532 & 0.950 & 0.130 & 0.026 & 0.006 & 0.001 \\
\hline 137 & 3.374 & 0.916 & 0.134 & 0.030 & 0.007 & 0.001 \\
\hline 138 & 2.366 & 0.664 & 0.119 & 0.033 & 0.008 & 0.001 \\
\hline 139 & 1.520 & 0.456 & 0.111 & 0.038 & 0.010 & 0.002 \\
\hline 140 & 1.006 & 0.336 & 0.113 & 0.044 & 0.011 & 0.002 \\
\hline 141 & 0.708 & 0.274 & 0.123 & 0.052 & 0.014 & 0.002 \\
\hline 142 & 0.529 & 0.247 & 0.139 & 0.062 & 0.016 & 0.003 \\
\hline 143 & 0.419 & 0.239 & 0.161 & 0.073 & 0.019 & 0.003 \\
\hline 144 & 0.397 & 0.356 & 0.314 & 0.154 & 0.043 & 0.008 \\
\hline 145 & 0.337 & 0.597 & 0.476 & 0.204 & 0.053 & 0.009 \\
\hline 146 & 0.430 & 0.742 & 0.572 & 0.241 & 0.062 & 0.011 \\
\hline 147 & 0.569 & 0.950 & 0.697 & 0.285 & 0.073 & 0.012 \\
\hline
\end{tabular}


Table III continued.

\begin{tabular}{|c|c|c|c|c|c|c|}
\hline \multirow{2}{*}{$\begin{array}{r}\text { Coupled } \\
\text { Bunch Mode }\end{array}$} & \multicolumn{6}{|c|}{ Growth Rate in $\mathrm{sec}^{-1}$} \\
\hline & $m=1$ & $m=2$ & $m=3$ & $m=4$ & $m=5$ & $m=6$ \\
\hline 148 & 0.801 & 1.287 & 0.885 & 0.345 & 0.086 & 0.015 \\
\hline 149 & 1.237 & 1.908 & 1.213 & 0.443 & 0.106 & 0.018 \\
\hline 150 & 2.103 & 3.129 & 1.836 & 0.621 & 0.141 & 0.023 \\
\hline 151 & 3.389 & 4.949 & 2.777 & 0.894 & 0.196 & 0.031 \\
\hline 152 & 3.427 & 5.125 & 3.071 & 1.069 & 0.250 & 0.042 \\
\hline 153 & 2.383 & 3.877 & 2.799 & 1.152 & 0.301 & 0.053 \\
\hline 154 & 1.718 & 3.080 & 2.621 & 1.203 & 0.332 & 0.060 \\
\hline 155 & 1.265 & 2.397 & 2.202 & 1.053 & 0.296 & 0.054 \\
\hline 156 & 0.893 & 1.721 & 1.616 & 0.781 & 0.221 & 0.041 \\
\hline 157 & 0.625 & 1.205 & 1.131 & 0.547 & 0.154 & 0.028 \\
\hline 158 & 0.257 & 0.601 & 0.687 & 0.361 & 0.106 & 0.020 \\
\hline \multicolumn{7}{|c|}{ Laudau Damping rate $\left(\mathrm{s}^{-1}\right)$} \\
\hline & 0.336 & 0.475 & 0.581 & 0.671 & 0.751 & 0.822 \\
\hline
\end{tabular}

\section{$2.1 \quad$ RESISTIVE WALL}

A most serious transverse coupled-bunch instability in a storage ring may be driven by the resistive wall. If there are $M_{s}$ identical equally spaced bunches in the ring, there are $\mu=0, \cdots, M_{s}-1$ transverse coupled modes when the center-of-mass of one bunch leads its predecessor by the betatron phase of $2 \pi \mu / M_{s}$. At the same time, each bunch can execute longitudinal motion with $m=0,1, \cdots$ nodes. The growth rate for the mode $\mu m$ is [4]

$$
\frac{1}{\tau_{\mu m}}=-\frac{1}{1+m} \frac{e M I_{b} c}{4 \pi \nu_{\beta} E} \sum_{k} \operatorname{Re} Z_{\perp}\left[\left(k M_{s}+\mu+\nu_{\beta}+m \nu_{s}\right) \omega_{0}\right] F_{m}^{\prime}\left(\omega \tau_{L}-\chi\right)
$$

where $M$ is the number of bunches. Strictly speaking Eq. (2.1) is correct only if $M=M_{s}$ or a completely filled ring. For example, in the $36 \times 36$ scenario with $1.7 \times 10^{11}$ particles per bunch and rms bunch length $60 \mathrm{~cm}$, the bunch spacing is 21 buckets; therefore $M=36$ and $M_{s}=H / 21=53$. On the other hand, in the $108 \times 108$ scenario with 7 -bucket spacing, $2.7 \times 10^{11}$ particles per bunch and rms bunch length $50 \mathrm{~cm}, M=108$ and $M_{s}=1113 / 7=159$. There are many unfilled buckets in both scenarios; thus Eq. (2.1) will not be an accurate description 


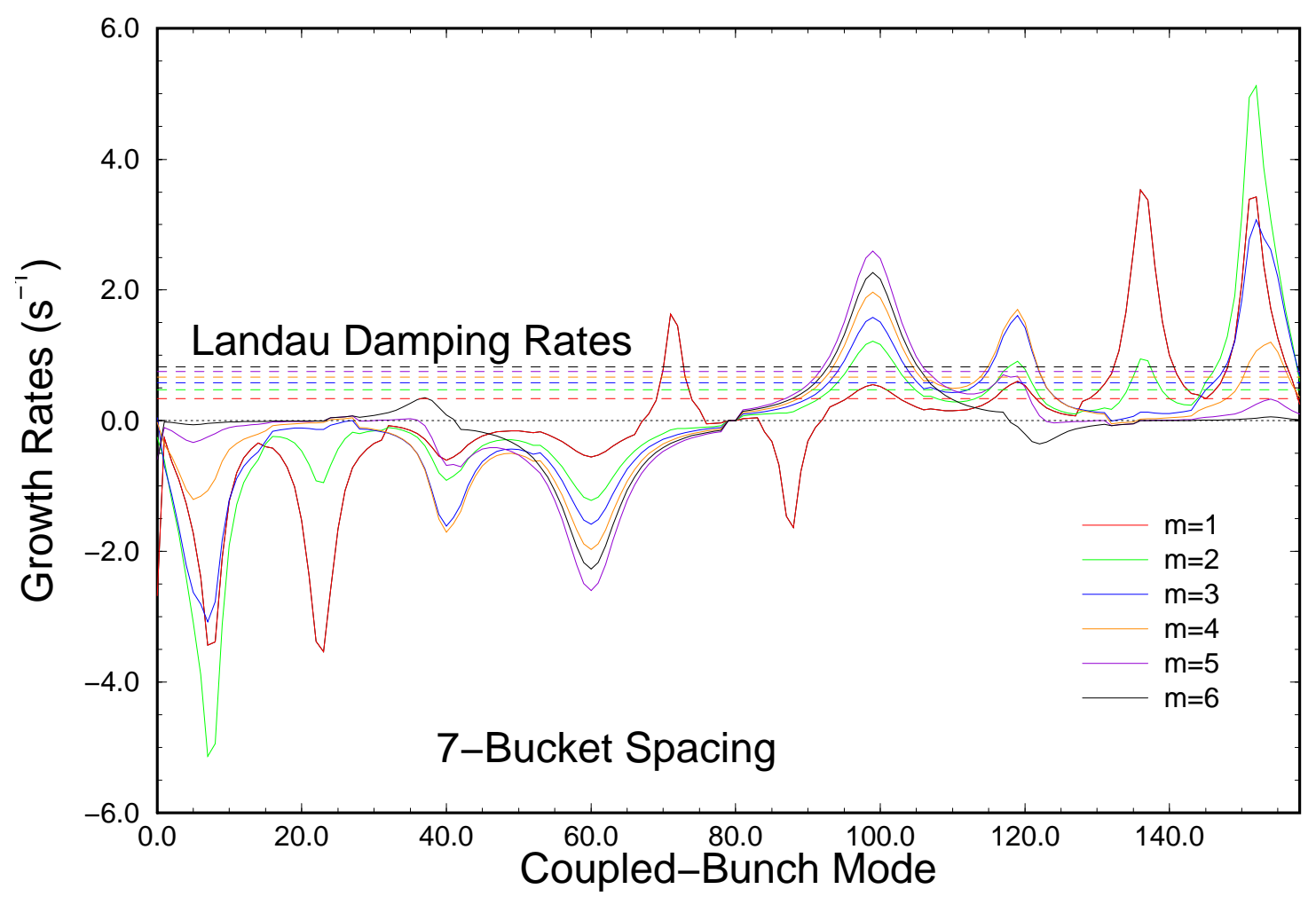

Figure 7: (color) Plot of growth rates (positive) and damping rates (negative) of each coupled-bunch mode driven by the higher-order parasitic resonances of the 8 Tevatron cavities. The bunch spacing is 7 buckets. The Landau damping rates are shown in dashes.

of the beam dynamics.

As the frequency $\omega \rightarrow \pm 0$, the real part of the resistive-wall impedance approaches first $\pm|\omega|^{-1 / 2}$, then $|\omega|^{-1}$ when the skin depth exceeds the thickness of the pipe wall, and finally zero when the frequency is exactly zero. At the residual betatron tune of the Tevatron, $\left[\nu_{\beta}\right] \sim 0.57$, we are in the regime of $\pm|\omega|^{-1 / 2}$ dependency. Therefore, there is always a mode $\mu$ that corresponds to a large negative $\mathcal{R} e Z_{\perp}$ and drives the transverse coupled-bunch instability. For example, with the betatron tune $\nu_{\beta}=20.57$, mode $\mu=53-21=32$ $(\mu=159-21=138$ for the 108 -by-108 scenario $)$ or frequency $-0.43 \omega_{0} /(2 \pi)$ with $k=0$ in the summation of Eq. (2.1) contributes the largest negative $\operatorname{Re} Z_{\perp}$, which is $-66.70 \mathrm{M} \Omega / \mathrm{m}$ according to our former estimate made in Ref. [5]. The next contribution with $k=1$ will give $\mathcal{R} e Z_{\perp}=+6.03 \mathrm{M} \Omega / \mathrm{m}$ in the $36 \times 36$ scenario and $+3.47 \mathrm{M} \Omega / \mathrm{m}$ for protons in the $108 \times 108$ scenario. The average current per bunch is $I_{b}=1.300 \mathrm{~mA}$ for the first scenario and $2.064 \mathrm{~mA}$ for the second. The growth rate is therefore given mostly by the $k=0$ term in the summation 


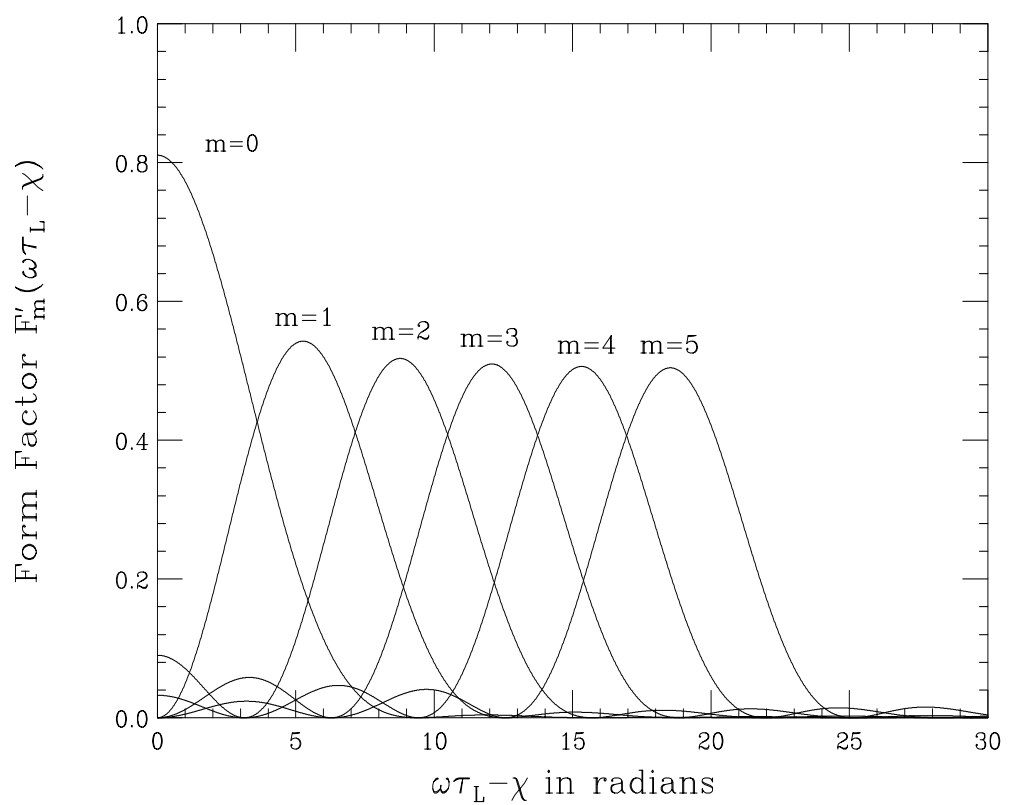

Figure 8: Plot of form factor $F_{m}^{\prime}\left(\omega \tau_{L}-\chi\right)$ for modes $m=0$ to 5 . With the normalization in Eq. (2.3), these are exactly the power spectra $h_{m}$.

and is very insensitive to the choice of $M_{s}$ in Eq. (2.1). For such a low driving frequency, only the lowest longitudinal mode $m=0$ will be excited. The growth rates after doing the actual summations are 19.5 and $93.2 \mathrm{~s}^{-1}$, respectively, for the two scenarios. Modes $\mu=31,30,29$, $\cdots(\mu=137,136,135, \cdots$ for the 108-by-108 scenario) are also unstable; the growth rates are, respectively, $10.6,8.0,6.6, \cdots \mathrm{s}^{-1}$, and $51.0,39.0,32.8, \cdots \mathrm{s}^{-1}$ for the two operating scenarios. The computation has been performed at zero chromaticity $(\xi=0)$, so that the chromatic phase $\chi=\xi \omega_{0} \tau_{L} / \eta=0$. Also, we have used the form factor $F_{0}^{\prime}(0)=8 / \pi^{2} \approx 0.811$, where, for convenience, Sacherer's sinusoidal modes of excitation have been assumed. These growth rates are much larger than those in Run I because there are more bunches. If one operates at chromaticity $\xi=+6, \chi=5.69$ and $F_{0}^{\prime}(5.69) \approx 0.142$ from Fig. 8 for the first scenario, while $\chi=4.75$ and $F_{0}^{\prime}(5.69) \approx 0.255$ for the second scenario. The growth rates for $\mu=32$ (or 138) drop to 3.42 and $29.3 \mathrm{~s}^{-1}$, respectively, which can be damped by a tune spread. For example, a tune spread of $\Delta \nu_{\beta}=0.0001$ will lead to a spread of betatron angular frequency of $\Delta \nu_{\beta} \omega_{0}=30 \mathrm{~s}^{-1}$, and will damp a growth rate up to $\sim 17.0 \mathrm{~s}^{-1}$ (FWHM for a Gaussian spread) [4]. For further discussion, we need to study the sinusoidal modes of excitation in the next subsection. 


\subsection{SINUSOIDAL MODES}

The Sacherer's sinusoidal modes of excitation consist of the orthonormal set

$$
p_{m}(\tau)= \begin{cases}\cos (m+1) \pi \frac{\tau}{\tau_{L}} & m=0,2, \cdots, \\ \sin (m+1) \pi \frac{\tau}{\tau_{L}} & m=1,3, \cdots,\end{cases}
$$

such that $p_{m}(\tau)$ has $m$ nodes along the bunch not including the ends. The power spectrum is proportional to

$$
h_{m}(\omega)=\frac{4(m+1)^{2}}{\pi^{2}} \frac{1+(-1)^{m} \cos \pi y}{\left[y^{2}-(m+1)^{2}\right]^{2}},
$$

where $y=\omega \tau_{L} / \pi$ and $\omega=k M_{s}-\mu+\nu_{\beta}+m \nu_{s}-\chi / \tau_{L}$. They are plotted in Fig. 9. The normalization of $h_{m}(\omega)$ in Eq. (2.3) has been chosen in such a way that, when the smooth approximation is applied to the summation over $k$, we have

$$
B \sum_{k=-\infty}^{+\infty} h_{m}(\omega) \approx \frac{B}{M_{s} \omega_{0}} \int_{-\infty}^{+\infty} h_{m}(\omega) d \omega=1
$$

Here $B=M_{s} \omega_{0} \tau_{L} /(2 \pi)$ is the bunching factor, or the ratio of full bunch length to bunch separation. Then the form factor $F_{m}^{\prime}(\omega)$ in Eq. (2.1) just equals $h_{m}(\omega)$.

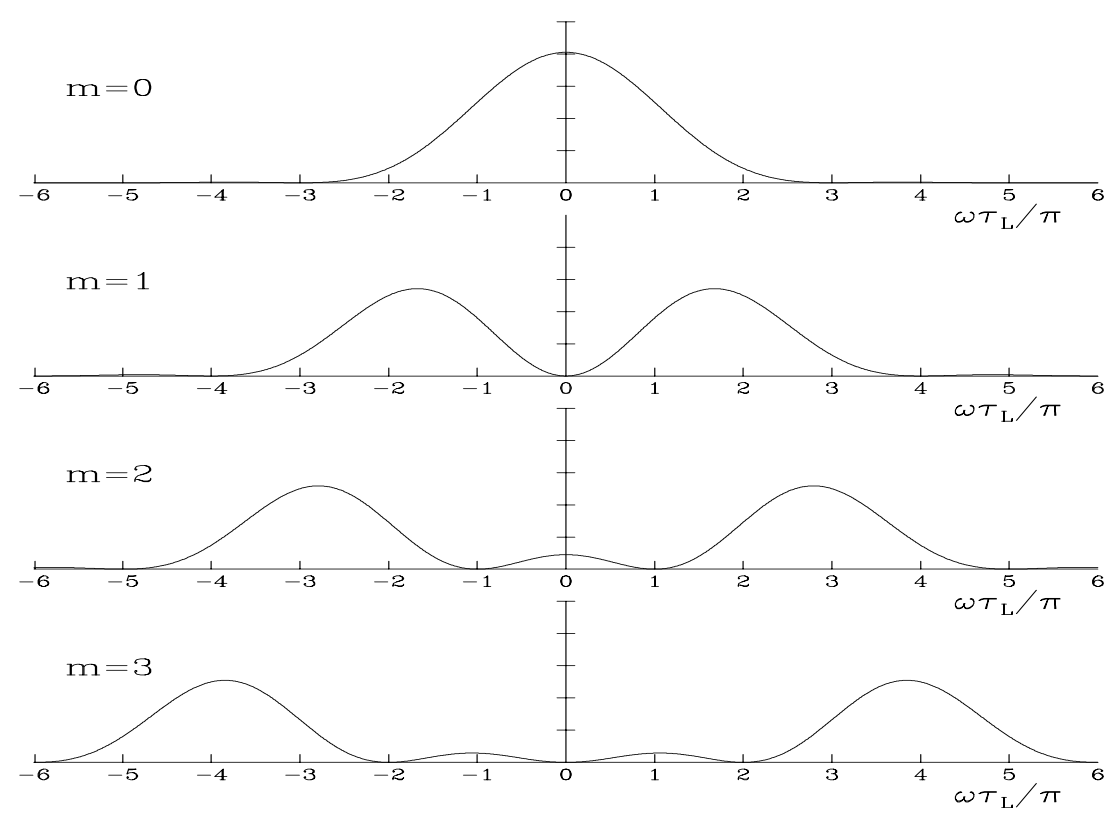

Figure 9: Power spectra $h_{m}(\omega)$ for modes $m=0$ to 3 with zero chromaticity. 
The Sacherer integral equation for transverse instability is an eigen-value-eigen-function problem when the unperturbed longitudinal distribution $g_{0}(r)$ in the longitudinal phase space is given. Physically, the modes of excitation $p_{m}(\tau)$ are the projection of the eigen-functions in the longitudinal phase space onto the time axis. The sinusoidal modes correspond to the water-bag distribution in phase space, so that the linear distribution is

$$
\rho(\tau) \propto \sqrt{\hat{\tau}^{2}-\tau^{2}} .
$$

For the distribution $g_{0}(r) \propto\left(\hat{\tau}^{2}-r^{2}\right)^{-1 / 2}$ in the longitudinal phase space, $p_{m}(\tau)$ are the Legendre polynomials and the Fourier transforms the spherical Bessel functions $j_{m}$. When $g_{0}(r)$ is bi-Gaussian, $p_{m}(\tau)$ are Hermite polynomials. Sometimes the growth rates computed are rather sensitive to the longitudinal bunch distribution assumed. Therefore, results in this section are estimates only.

We now learn that a chromaticity of $\xi=\eta /\left(f_{0} \tau_{L}\right)=+10.73$ will push the power spectra in Fig. 9 to the right (or positive frequency side) by two $\omega \tau_{L} / \pi$ units. The $m=0$ will then only see the positive-frequency impedance and no instability will result. However, the $m=1$ mode will now peak at zero frequency and the resistive wall impedance will drive the $m=1$ mode unstable and a quadrupole transverse damper will be required, if Landau damping coming from tune spread is not large enough.

\subsection{TRANSVERSE COUPLED-BUNCH INSTABILITY DRIVEN BY RESONANCES}

The narrow transverse resonant modes of the rf cavities will also drive transverse coupledbunch instability. The growth rate is described by the general growth formula of Eq. (2.1). When the resonance is narrow enough, only one frequency $-\omega_{r} /(2 \pi)$ contributes in the summation. Thus the growth rate becomes

$$
\frac{1}{\tau_{\mu m}}=-\frac{1}{1+m} \frac{e M I_{b} c}{4 \pi \nu_{\beta} E} \mathcal{R e} Z_{\perp}\left(\omega_{r}\right) F_{m}^{\prime}\left(\omega_{r} \tau_{L}-\chi\right)
$$

where $M$ is the number of bunches and the frequency $\omega_{r}$ is negative.

There have not been any measurements for the dipole modes in the rf cavities, and we need to rely on the URMEL results, which are listed in Table IV. The contribution of all the resonances to the 53 coupled-bunch modes in the 36-by-36 scenario is shown in Fig. 10. 
Table IV: Transverse modes for one whole cavity.

\begin{tabular}{lcrc}
\hline Mode Type & $\begin{array}{c}\text { Frequency } \\
(\mathrm{MHz})\end{array}$ & $\begin{array}{c}R / Q \\
(\Omega / \mathrm{m})\end{array}$ & $Q$ \\
\hline 1-EE-1 & 486.488 & 229.80 & 31605 \\
1-ME-2 & 486.864 & 148.95 & 31487 \\
1-EE-2 & 513.370 & 117.38 & 33262 \\
1-ME-3 & 518.317 & 117.93 & 34008 \\
1-EE-3 & 561.727 & 81.62 & 33029 \\
1-ME-4 & 575.298 & 3.84 & 35810 \\
1-EE-4 & 625.123 & 61.00 & 32598 \\
1-ME-5 & 650.853 & 35.21 & 37592 \\
1-EE-5 & 699.723 & 54.76 & 33407 \\
\hline
\end{tabular}

Because we are looking at negative frequencies, the real part of the transverse impedance is negative. The upper trace is for one cavity while the lower trace for 8 cavities again assuming 8 -fold de-Quing. Together with each coupled-bunch mode $\mu$, certain longitudinal azimuthal modes $m$ will be excited and transverse oscillations along the bunch will be observed. These azimuthal modes are usually called head-tail modes. As it will be shown later that the growth rates for various modes are small, we do not compute the growth rates for all the modes. Instead, we compute only the fastest growing modes excited by each resonance. The results are listed in Table $\mathrm{V}$ for the 36 -by-36 scenario.

Some comments are in order. Here, we assume that the higher-order modes of the $8 \mathrm{rf}$ cavities do not fall on top of each other at exactly the same frequencies. Instead, we assume that the resonances summed over 8 cavities will be de-Qued 8 times and the shunt impedance corresponding to a certain resonance will be the same as that for a single cavity. At the same time, we assume that a peak of a resonance, when summed over the $8 \mathrm{rf}$ cavities, falls exactly on top of a particular synchrotron sideband of the betatron line. ${ }^{\S}$ The frequencies of the lowest 9 higher-order modes range from 486.5 to $699.7 \mathrm{MHz}$. Therefore $\omega_{r} \tau_{L} / \pi-\chi / \pi\left(\omega_{r}\right.$ is negative) for the 36 -by-36 scenario ranges from -8.7 to -12.5 at zero chromaticity. From the power spectra in Fig. 9, this implies modes roughly from $m=8$ to 11 will be excited. These are listed in column 4 of the table. We can see in Table $\mathrm{V}$ that the growth rates actually peak for these modes. Since the growth rates are affected so much by the mode of excitation, we also give the bare growth rate for each resonance in column 5 when the form factor $F_{m}^{\prime}$ and

\footnotetext{
${ }^{\S}$ This assures the fastest growth rates.
} 


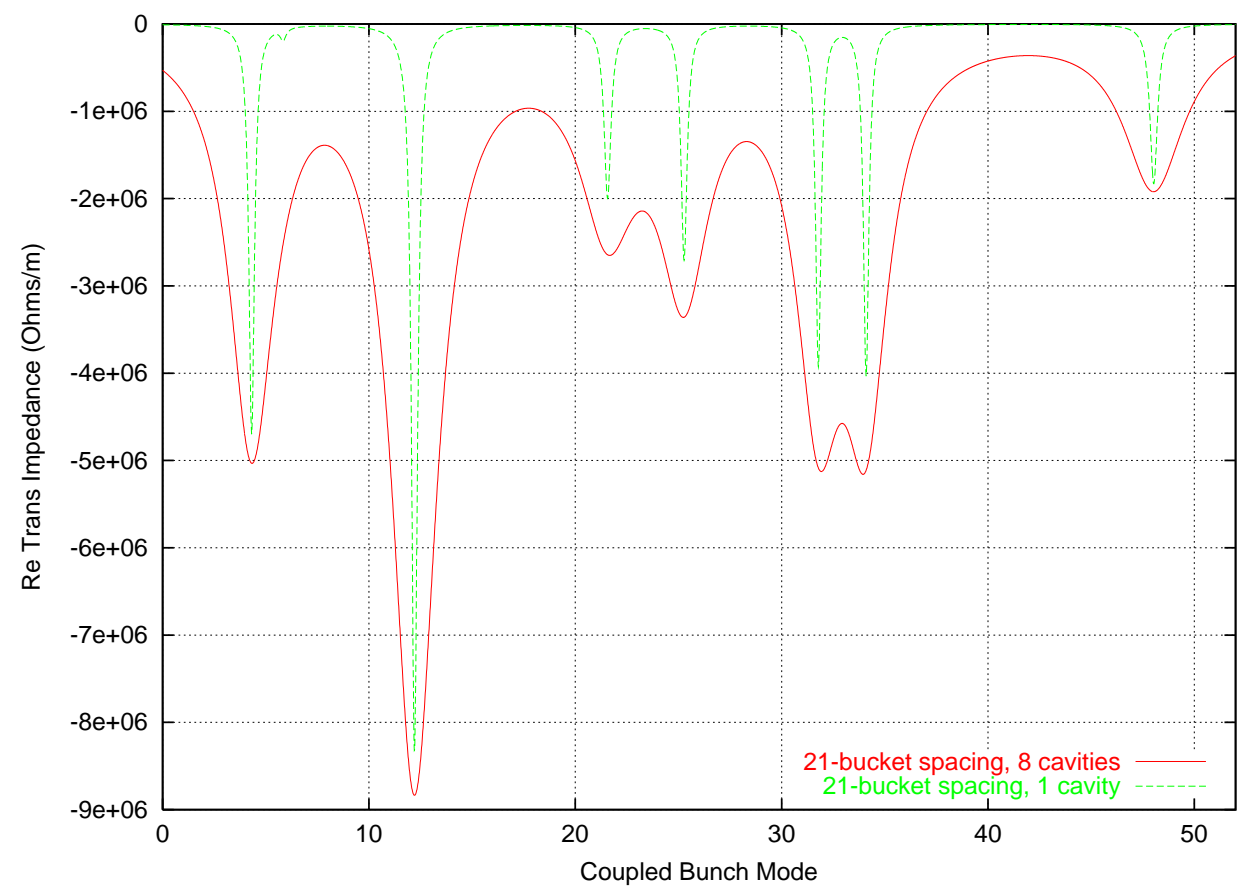

Figure 10: (color) The real part of the transverse impedance of the higher-order dipole resonances are plotted as a function of the coupled-bunch mode $\mu$ that they will drive. The upper (green) trace is for one cavity while the lower (red) trace corresponds to 8 cavities assuming that each resonance spreads out 8 folds. The bunch spacing is 21 buckets.

Table V: Growth rates for transverse coupled-bunch modes driven by higher-order dipole modes of the rf cavities in the $36 \times 36$ scenario. The fastest growth rate driven by each higher-order dipole mode is underlined.

\begin{tabular}{|c|c|c|c|c|c|c|c|c|c|c|c|c|}
\hline \multirow{2}{*}{$\begin{array}{c}f_{r} \\
\mathrm{MHz}\end{array}$} & \multirow{2}{*}{$\begin{array}{r}R_{s} \\
\Omega / m\end{array}$} & \multirow[t]{2}{*}{$Q$} & \multirow[t]{2}{*}{$m_{\mathrm{pk}}$} & \multicolumn{8}{|c|}{ Growth Rate $\left(\mathrm{s}^{-1}\right)$} & \multirow{2}{*}{$\begin{array}{c}\text { Coupled } \\
\text { Mode } \\
\mu\end{array}$} \\
\hline & & & & $\mathrm{h}$ & $m=5$ & $m=6$ & $m=7$ & $m=8$ & $m=9$ & & & \\
\hline \multicolumn{13}{|c|}{ Chromaticity $\xi=0$} \\
\hline 486.5 & 7262 & 31605 & 7 & 7 & 06 & 0.004 & 0.098 & $\underline{0.141}$ & 0.029 & 02 & 04 & 12 \\
\hline 486.9 & 4689 & 31487 & 7.7 & 1.696 & 0.004 & 0.002 & 0.063 & $\underline{0.091}$ & 0.019 & 0.001 & 0.003 & 4 \\
\hline 513.4 & 3904 & 33262 & 8.2 & 1.412 & 0.003 & 0.001 & 0.020 & $\underline{0.075}$ & 0.043 & 0.001 & 0.004 & 32 \\
\hline 518.3 & 4010 & 34008 & 8. & 1 & 2 & 0.001 & 0.016 & $\underline{0.073}$ & 0.050 & 02 & 03 & 34 \\
\hline 561.7 & 2695 & 33029 & 5 & 0 & 0 & 0.002 & 0.000 & 0. & $\underline{0.048}$ & 22 & 00 & 25 \\
\hline 575.3 & 137 & 358 & 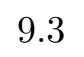 & 0 & 0 & 0.000 & 0.0 & 0.000 & 0.002 & 0.002 & 0 & 6 \\
\hline 625.1 & 1988 & 32598 & 10.2 & 0.719 & 0.000 & 0.000 & 0.001 & 0.000 & 0.008 & $\underline{0.031}$ & 0.018 & 22 \\
\hline 650.9 & 1323 & 37592 & 10.7 & 0.479 & 0.000 & 0.000 & 0.000 & 0.001 & 0.001 & 0.014 & $\underline{0.019}$ & 12 \\
\hline 699.7 & 1829 & 33407 & 11.5 & 0.662 & 0.000 & 0.000 & 0.000 & 0.000 & 0.001 & 0.002 & $\underline{0.021}$ & 48 \\
\hline
\end{tabular}


the factor $(1+m)^{-1}$ are not included. Increasing the chromaticity shifts the mode spectra of the bunch to the right (positive frequency side); so head-tail modes of much higher $m$ will be excited. On the other hand, running the Tevatron at negative chromaticities will shift the bunch mode spectra to the right. and head-tail modes with lower $m$ will be excited instead. As a whole, the growth rates are extremely slow. A tune spread of $\Delta \nu_{\beta}=0.0001$, for example, will damp a growth rate up to $\sim 17 \mathrm{~s}^{-1}$.

We next look at the 108-by-108 scenario. For the symmetrically filled ring with 7-bucket spacing, there are 159 coupled-bunch modes. The real parts of the transverse impedance contributing to these modes are depicted in Fig. 11. The upper trace is for one cavity while the lower trace for 8 cavities again assuming 8-fold de-Quing. Again, we compute only the fastest growing modes driven by individual resonances. The results are listed in Table VI.

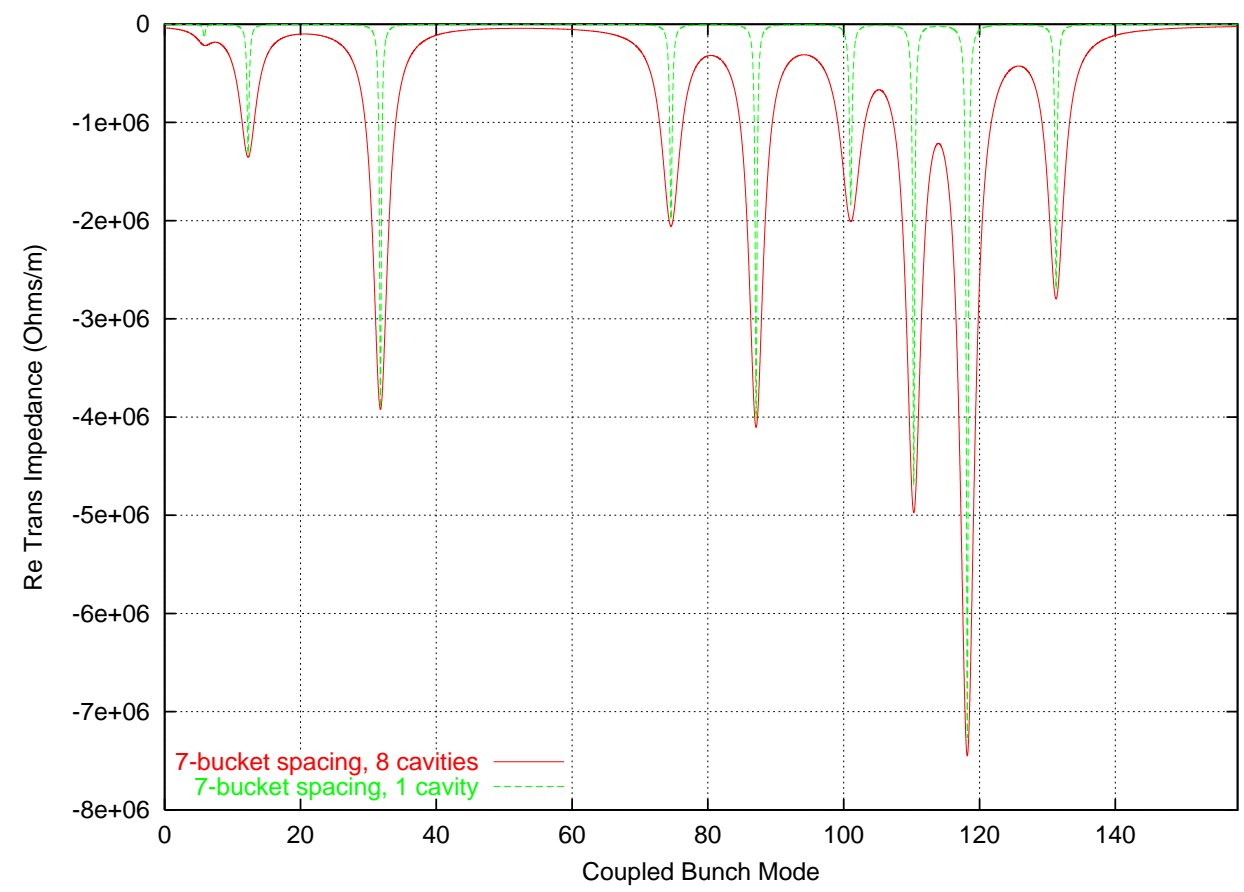

Figure 11: (color) The real part of the transverse impedance of the higher-order dipole resonances are plotted as a function of the coupled-bunch mode $\mu$ that they will drive. The upper (green) trace is for one cavity while the lower (red) trace corresponds to 8 cavities assuming that each resonance spreads out 8 folds. The bunch spacing is 21 buckets.

In this scenario, because of the shorter bunch length $\left|\omega_{r} \tau_{L} / \pi\right|$ will be smaller and modes $m=6$ to 9 will be driven instead. These are shown in Table VI. Due to the presence of more bunches, high bunch intensity, and short bunch length, the growth rates are relatively faster 
Table VI: Growth rates for transverse coupled-bunch modes driven by higher-order dipole modes of the rf cavities in the $108 \times 108$ scenario. The fastest growth rate driven by each higher-order dipole mode is underlined.

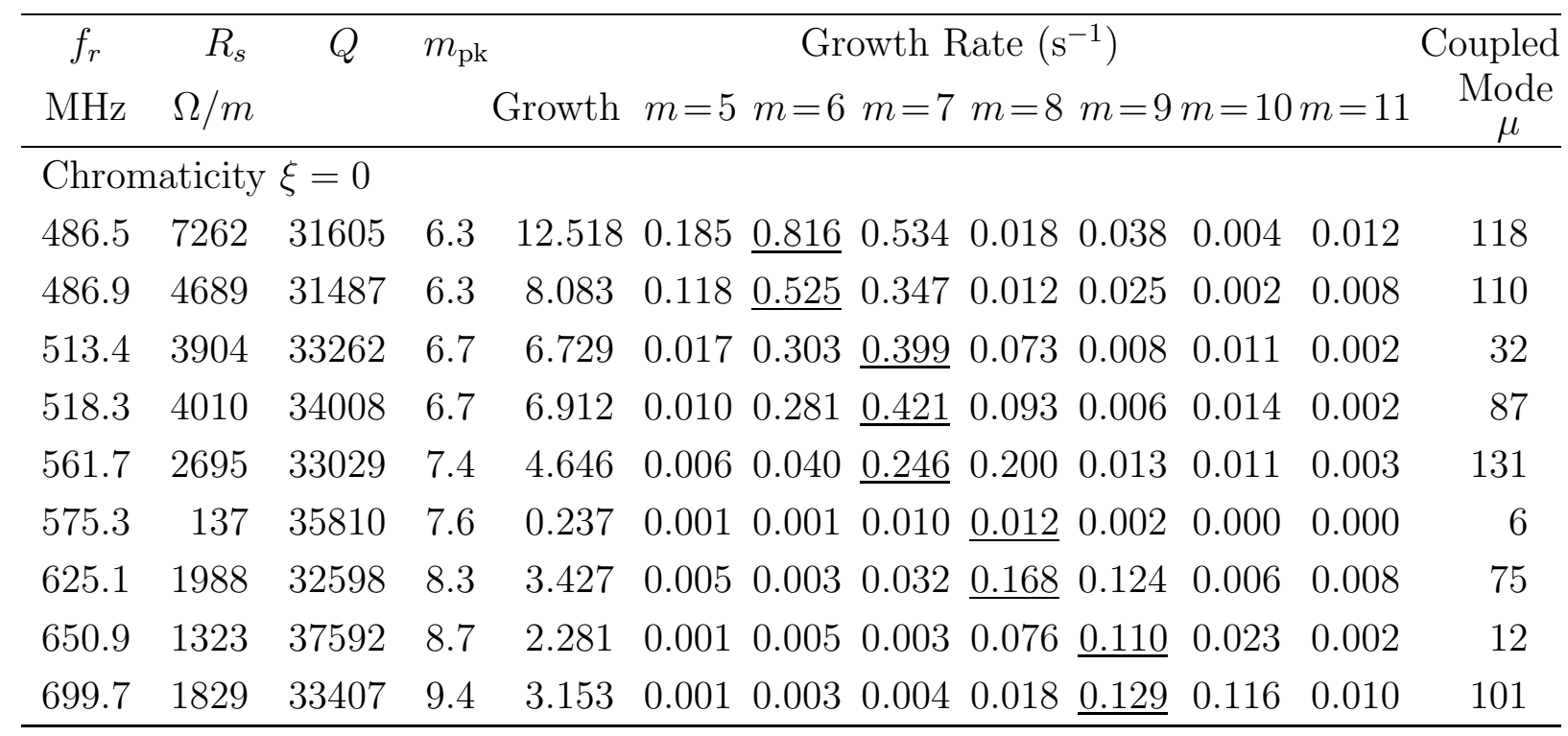

than those for the 36 -by-36 scenario. The fastest growth rate shown is $0.816 \mathrm{~s}^{-1}$, which will be damped by a small betatron tune spread. As a whole, transverse coupled-bunch instabilities driven by the higher-order modes in the rf cavities should not represent a problem at all.

\section{References}

[1] F.J. Sacherer, IEEE Trans. Nuclear Sci. NS 20, 3, 825 (1973).

[2] D. Sun, private communication.

[3] See for example, K.L.F. Bane, P.B. Wilson, and T. Weiland, AIP Proc. 127, Phys. of High Energy Accel., BNL/SUNY, 1983, p.875.

[4] F.J. Sacherer, Theoretical Aspects of the Behaviour of Beams in Accelerators and Storage Rings, Proc. First Course of Int. School of Part. Accel., Erice, Nov. 10-22, 1976, p.198.

[5] K.Y. Ng, Impedances and Collective Instabilities of the Tevatron at Run II, Fermilab Report TM-2055, 1998. 